\title{
Lemierre's Syndrome: A Case of a Large Inflammatory Neck Mass with Internal Jugular Vein Thrombosis and Literature Review of Its Surgical Intervention
}

\section{Yilin Shen}

Shanghai Jiao Tong University Medical School Affiliated Ruijin Hospital

\section{Bin Ye}

Shanghai Jiao Tong University Medical School Affiliated Ruijin Hospital

\section{Qian Da}

Shanghai Jiao Tong University Medical School Affiliated Ruijin Hospital

\section{Chaofu Wang}

Shanghai Jiao Tong University Medical School Affiliated Ruijin Hospital

Mingliang Xiang ( $\sim$ Mingliangxiang@163.com)

Shanghai Jiao Tong University Medical School Affiliated Ruijin Hospital https://orcid.org/0000-00019253-9852

\section{Case report}

Keywords: Head and neck infection, Internal jugular vein thrombosis, Lemierre's syndrome, Surgical treatment

Posted Date: June 7th, 2021

DOI: https://doi.org/10.21203/rs.3.rs-543943/v1

License: (c) (i) This work is licensed under a Creative Commons Attribution 4.0 International License. Read Full License 


\section{Abstract}

Background: Lemierre's syndrome is a fatal and rare disease that is typically characterized by oropharyngeal infection and internal jugular vein thrombosis. Timely application of appropriate antibiotics is the standard treatment. However, when conservative medical treatment fails to have effect, surgical intervention may be the only effective option for controlling the source of infection.

Case presentation: The authors report a case of Lemierre's syndrome with a large inflammatory neck mass involving left internal jugular vein thrombosis that was cured by surgical treatment. In addition, a literature review was carried out through PubMed using the terms "Lemierre's syndrome/disease and review, meta-analysis or retrospective study" and "Lemierre's syndrome/disease and internal jugular vein". This search yielded 6 articles that recorded the surgical rate in detail.

Conclusion: The purpose of this review was to summarize the conditions under which surgical treatment are conducted. Additionally, this is the first report of such a large inflammatory neck mass that was completely cured by surgical resection and internal jugular vein ligation. The authors further offer several conclusions regarding surgical intervention in Lemierre's syndrome.

\section{Background}

Internal jugular vein (IJV) thrombosis is a relatively rare and urgent disease. In a retrospective study, the number of such cases occurring from 2001 to 2008 was 2.5 times that occurring from 1991 to 2000 and 20 times that occurring from 1980 to 1990 [1]. The reasons behind the increasing incidence of IJV thrombosis include the advancement of antibiotic resistance, widespread use of hemodialysis, general application of central venous catheters, expansion of assisted reproductive technology, and increasing incidence of cancer [2]. In a nine-year retrospective study of 1,948 patients with deep vein thrombosis, only 29 patients developed IJV thrombosis, of whom 23 had IJV thrombosis secondary to another condition, such as a malignancy (e.g., Trousseau syndrome), central venous catheter implantation, or ovarian hyperstimulation syndrome (OHSS) [3]. In addition, bilateral internal jugular vein thrombosis is an important indicator of malignant tumors. In a 5-year retrospective study of 41 patients with IJV thrombosis in Germany, paraneoplastic thrombosis accounted for $54 \%$ of cases; of these cases, otolaryngology head and neck diseases accounted for $68 \%$. The other patients mostly had inflammatory diseases [4].

In the ear, nose and throat (ENT) field, IJV thrombosis is commonly associated with Lemierre's syndrome (LS), which is a complication of infectious diseases, such as otitis media and oropharyngeal abscess or infection. LS is commonly defined by the following diagnostic criteria: 1) oropharyngeal infection; 2) internal jugular vein thrombophlebitis or thrombosis; 3) septic emboli at a remote site, more frequently the lungs; and 4) isolation of Fusobacterium nucleatum on blood culture[5]. LS is usually accompanied by septic emboli in the lungs or other organs[6]. Under some rare conditions, LS can also be triggered by tooth extraction[7]. Pulmonary embolism, with an incidence of approximately $10 \%$, and postthrombotic 
syndromes, such as limb pain, heaviness, venous dilatation, edema, pigmentation, nutritional skin changes, and venous ulcers, are complications of IJV thrombosis[4]. Therefore, ENT doctors should give enough attention to patients with IJV thrombosis to avoid the disastrous results caused by pulmonary or cerebral thrombosis.

To the best of our knowledge, this is the first case of such a large infectious neck mass with internal jugular vein thrombosis that was completely cured by surgical intervention.

\section{Case Presentation}

A 67-year-old male developed pain in the left neck 14 days prior after eating mud fish. He was healthy and denied a history of infectious diseases, chronic diseases, surgical trauma, blood transfusion, allergies or contact with poisonous substances. Physical examination on admission revealed the following: fever, chills, fatigue, mild dysuria, diffusive swelling pain of the neck on the left side, and high skin temperature. His left neck was tender and edematous with cellulitis. The mass was scleroid with a liquefied center. Other parameters were as follows: white blood cell (WBC) count, $12.2^{\star} 10^{9} / \mathrm{L}$; neutrophil count, $11.76 * 10^{9} / \mathrm{L}$; platelet (PLT) count, $51 * 10^{9} / \mathrm{L}$; C-reactive protein (CRP) level, $146 \mathrm{mg} / \mathrm{L}$; procalcitonin (PCT), $156.99 \mathrm{ng} / \mathrm{ml}$; temperature, $39.5^{\circ} \mathrm{C}$; respiratory rate, $24 /$ minute; pulse, $118 /$ minute; and blood pressure (BP), $109 / 61 \mathrm{mmHg}$. This patient stated that he had been to many hospitals and that antibiotics like cephalosporin slightly alleviated his neck pain at first. However, the effect was temporary and no longer present after he transferred to Shanghai. Three days before he presented to our hospital, he felt extreme swelling and pain again in the area of his neck mass (Fig. 1). A series of further examinations were performed, with the following results: glucose, $22.13 \mathrm{mmol} / \mathrm{L}$; APTT, $43.1 \mathrm{~s} ; \mathrm{PT}, 17.3 \mathrm{~s} ; \mathrm{Fg}, 5.0 \mathrm{~g} / \mathrm{L}$; FDP, $7.3 \mathrm{mg} / \mathrm{L}$; and D-dimer (D-D), $2.1 \mathrm{mg} / \mathrm{L}$. Urine analysis was positive for glucose, blood, protein, and white blood cells. Ultrasound revealed mixed echogenicity in the left neck mass that was irregular in shape. The mass was approximately $74 * 37 \mathrm{~mm}$ in size. Color Doppler revealed generalized thrombosis of the internal jugular vein. CT of the chest and neck was conducted and suggested that the cervical abscess extended to the thorax and superior mediastinum, without a signal from the left internal jugular vein. Multiple enlarged cervical lymph nodes were observed. The trachea and left thyroid were also compressed (Fig. 2). Video laryngoscopy excluded the possibility of pyriform sinus fistula or any foreign body. Pus obtained from the mass showed many neutrophils and large amounts of necrotic tissue. No microbes could be isolated from the samples (blood and pus). The infectious disease and nephrology departments were consulted. Fourteen days of combination antibiotic treatment and regular insulin treatment returned the patient's temperature, routine blood markers, CRP and PCT levels and coagulation function to normal. However, the neck mass remained. Therefore, the surgery department was consulted.

The patient was taken to the operating room. The neck mass adhered tightly to the surrounding tissue, and the involved segment of the left internal jugular vein was exposed by sharp dissection (Fig. 3). The proximal part of the mass needed to be ligated first to avoid small thrombus detachment. There was a large amount of inflammation and fibrosis present in the involved area. The involved segment of the internal jugular vein and the whole neck mass were completely resected, and the distal part of the internal 
jugular vein was fully ligated (Fig. 4). The pathological examination showed hemorrhagic necrosis with the proliferation of fibrous and granulation tissue and the accumulation of foam-like cells and multinuclear giant cells (Fig. 5).

\section{Literature review}

It is rare that patients with Lemierre's syndrome require surgical intervention when antibiotics and anticoagulant therapies fail. This is the first study to summarize cases of LS requiring surgical treatment (not including abscess drainage). First, we decided to collect previous reviews and meta-analyses to obtain good knowledge of the rate of surgery in LS. Search strategy and selection criteria: A search of the literature in MEDLINE was performed through PubMed to identify relevant English language articles. The following search terms were used: "Lemierre's syndrome/disease and review, meta-analysis or retrospective study" and "Lemierre's syndrome/disease and internal jugular vein". The references of the retrieved articles were also reviewed to identify additional sources. Through reading the abstract and the full text, we found a total of 6 reviews and meta-analyses (Table 1) that included detailed descriptions of patients who underwent surgery (not including abscess drainage). In a retrospective review from 19982010 at a local tertiary referral hospital, 17 of the 23 patients underwent surgical treatment of the primary infection site [8]. In a five-year systematic review, surgical procedures, such as tooth extraction, craniotomy and ligation of the occluded vein, were performed in 5 patients to prevent further septic emboli[9]. A retrospective study from June 2000 to May 2016 showed that IJV ligation was performed in only one of five LS cases at the Children's Hospital of Alabama[10]. In an 8-year Swedish nationwide retrospective study, three patients with peritonsillitis were surgically treated by tonsillectomy[11]. In the latest meta-analysis of 394 patients in 2020, only 10 patients underwent IJV ligation/excision, only 1 patient underwent ligation/excision of the thrombosed external carotid artery, 3 underwent endoscopic sinus surgery and 11 underwent mastoidectomy[12]. In the latest systematic review, which included the most LS cases reported, surgical procedures were performed in 101 patients, and 31 patients underwent IJV ligation/embolectomy[13].

Table 1

Rate of surgery in LS in previous reviews or meta-analyses.

\begin{tabular}{|lllll|}
\hline Author & Type & Year & Rate of surgery & Rate of IJV ligation \\
\hline Schubert[8] & Retrospective study & 2014 & $17 / 23$ & No description \\
\hline Johannesen et al[9] & Systematic review & 2016 & $5 / 137$ & $3 / 137$ \\
\hline Jariwala et al [10] & Retrospective study & 2017 & $1 / 7$ & $1 / 7$ \\
\hline Nygren et al[11] & Retrospective study & 2019 & $3 / 104$ & No description \\
\hline Gore[12] & Meta-analysis & 2020 & $25 / 394$ & $10 / 394$ \\
\hline Valerio[13] & Systematic review & 2021 & $101 / 652$ & $31 / 652$ \\
\hline
\end{tabular}




\section{Discussion}

In this study, we report a case of Lemierre's syndrome in an elderly male caused by an acute infectious neck mass. Timely comprehensive medical and surgical treatments were given to avoid serious complications.

Internal jugular vein thrombosis is a rare and serious emergent disease that needs to be identified early in the course, as it can lead to catastrophic consequences, such as stroke or pulmonary embolism. The main pathological basis of internal jugular vein thrombosis is as follows: (1) injury of venous intima; (2) slowing down of blood flow; and (3) hypercoagulability. The common causes are as follows [14-16]: (1) facial infection, such as furuncle and carbuncle, sinusitis, otitis media, and suppurative tonsillitis; bacteria can spread through the damaged mucosa and parapharyngeal space or invade the jugular vein through the lymphatic and venous systems, leading to infectious phlebitis and bacterial embolism information; (2) long duration of internal jugular vein catheterization; (3) head and neck surgery; (4) head and neck tumor; (5) pulmonary embolism; and (6) other systemic diseases, such as polycythemia. Doctors need to take care of patients immediately when encountering such cases.

Lemierre's syndrome can show the typical symptoms and signs of progressive infection, including sore throat, fever or neck pain. A systematic review of Lemierre's syndrome by Peter et al. found that in 84 patients, the most common first clinical presentation was a sore throat (33\%), followed by a neck mass (23\%) and neck pain (20\%)[1]. In the current case, the patient presented with fever and neck pain at first, followed by a neck mass. The use of antibiotics before he was transferred to our hospital was ineffective. The white blood cell count, PCT level, and ESR were elevated, and blood appeared in his urine. We adjusted the treatment to the combined application of broad-spectrum antibiotics, including imipenem and teicoplanin, for another 2 weeks. The patient's body temperature returned to normal and laboratory testing showed that the patient's infectious condition had been controlled, but the neck mass and internal jugular vein thrombosis persisted and required surgical treatment.

Fusobacterium necrophorum is the main pathogen of Lemierre's syndrome[1]. However, in this case, the culture of both blood and fluid obtained from the mass was negative for bacteria, which might be because the patient had been treated with antibiotics for nearly 2 weeks before coming to our hospital. This also suggests that it is particularly important for doctors to culture blood or fluid from the mass before the use of antibiotics in these patients.

The diagnosis of internal jugular vein thrombosis in Lemierre's syndrome relies on imaging examination. Ultrasound is the first choice for the diagnosis of LS, and computed tomography (CT) and magnetic resonance imaging (MRI) are currently implemented in general practice when necessary. Albertyn et al. first summarized the classic imaging features of internal jugular vein thrombosis. Ultrasonography shows the vein to be distended and nonpulsatile, with internal echoes. CT shows swelling of the adjacent soft tissues, distension of the vein with wall enhancement, and low-attenuation intraluminal filling defects. However, ultrasound has limitations and cannot display the anatomy behind the clavicle or mandible[16]. In this case, we found that although US can clearly show internal jugular vein thrombosis, 
knowing its boundaries and connection with the tumor still depends on CT or MRI, especially when there is an urgent need for surgery. A full assessment by preoperative imaging is of great importance. This is also consistent with the views of Charles et al[17].

Priority treatment for LS includes antibiotic therapy and drainage of the infected site. Rarely, other surgical procedures, such as ligation of the occluded vein, craniotomy and tooth extraction, are performed. Antithrombotic therapy, including novel oral anticoagulants (DOACs), is also recommended depending on the individual's condition. However, it remains controversial whether anticoagulation or antithrombotics are effective in Lemierre's syndrome. Some scholars think that thrombosis is due to the infection process and can be resolved when the infection has been controlled [18]. In this case, we did not immediately apply anticoagulant or thrombolytic therapies considering that the APTT of this patient was significantly prolonged at the time he came to our hospital and the consumption of platelets was relatively high; emergency anticoagulant therapy may have increased his risk of bleeding. To date, there have been no sufficient clinical studies and is no sufficient evidence suggesting the necessity for anticoagulant therapy in Lemierre's syndrome[19]. Previous studies have also reported the occurrence of extensive suppurative thrombophlebitis of the bilateral IJV and superior vena cava in patients with Lemierre's syndrome despite the use of antibiotics and anticoagulant therapy; adjunctive catheter-directed thrombolysis and superior vena cava stenting were performed to help these patients completely recover[20]. Anticoagulation therapy has not been shown to reduce the complications of Lemierre's syndrome, such as sepsis[17]. Meanwhile, Johannesen et al. did not find that anticoagulation therapy decreased the mortality rate or course of the disease or reduced the duration of antibiotic use[21]. However, anticoagulation therapy is recommended in patients with a poor clinical response despite antibiotic therapy and with a high risk of intracranial thrombosis or recurrent thrombophlebitis[22-24]. In this case, the cause of internal jugular vein thrombosis was largely infection, so surgical treatment was the best choice when antibiotics could not completely cure the infection and thrombosis. Through previous retrospective studies, systematic reviews and meta-analyses obtained by database searches, we summarized the following points regarding surgical intervention in Lemierre's syndrome:

(1) When patients do not respond to conservative medical therapy and continue to show extensive septic thrombosis or uncontrolled severe sepsis, surgical treatments need to be considered.

(2) Abscess drainage is the most common and convenient surgical treatment for abscesses upon formation.

(3) Surgical treatment of the primary infection site is effective for controlling the spread of infection and sepsis.

(4) IJV ligation or excision is suitable for patients with persistent septic embolization after treatment with antibiotics and anticoagulants.

(5) IJV ligation or excision is also appropriate to avoid thrombus detachment when anticoagulation therapy or catheter-directed thrombolysis is ineffective. 


\section{Conclusion}

Lemierre's syndrome is an extremely rare disease, but the fatality rate can reach $15 \%$, even with escalating antibiotic therapy[20]. Therefore, early diagnosis is particularly important, and the timely application of appropriate antibiotics is the standard treatment. Surgical intervention may be the only effective option for controlling the source of infection or when conservative medical treatment fails.

\section{Abbreviations}

Internal jugular vein: IJV

Ear, nose and throat: ENT

Lemierre's syndrome: LS

White blood cell: WBC

Platelet: PLT

C-reactive protein: CRP

Procalcitonin: PCT

Blood pressure: BP

Computed tomography: CT Magnetic resonance imaging: MRI

\section{Declarations}

\section{Ethics approval and consent to participate}

The studies involving human participants were reviewed and approved by Human Ethics Committee, Ruijin Hospital, Shanghai Jiao Tong University School of Medicine. The patient provided their written informed consent for the use of his images and other clinical information in this study. A copy of the written consent is available for review by the Editor-in-Chief of this journal.

\section{Consent for publication}

Not applicable.

\section{Availability of data and materials}

Not applicable.

Competing interest 
There are no conflicts of interest.

\section{Funding}

Not applicable.

\section{Authors' contributions}

Minglinag Xiang did the whole operation and checked review. Yilin Shen and Bin Ye have wrote article and made review. Chaofu Wang and Qian Da provided the pathological images.

\section{Acknowledgements}

The authors would like to thank the World Jouranl of Emergency Surgery.

\section{References}

1. Karkos PD, Asrani S, Karkos CD, Leong SC, Theochari EG, Alexopoulou TD, Assimakopoulos AD. Lemierre's syndrome: A systematic review. LARYNGOSCOPE. 2009;119(8):1552-9.

2. Leibman $Y$, Ayalon M, Steiner IP. Internal jugular venous thrombosis after in vitro fertilization. J EMERG MED. 2009;37(1):29-31.

3. Gbaguidi X, Janvresse A, Benichou J, Cailleux N, Levesque H, Marie I. Internal jugular vein thrombosis: outcome and risk factors. QJM. 2011;104(3):209-19.

4. Hahn J, Nordmann-Kleiner M, Hoffmann TK, Greve J. Thrombosis of the internal jugular vein in the ENT-department - Prevalence, causes and therapy: A retrospective analysis. AURIS NASUS LARYNX. 2019;46(4):624-9.

5. Kuppalli K, Livorsi D, Talati NJ, Osborn M. Lemierre's syndrome due to Fusobacterium necrophorum. LANCET INFECT DIS. 2012;12(10):808-15.

6. Yombi JC, Bogaert T, Tribak K, Danse E. Lemierre Syndrome of the Femoral Vein, Related to Fusobacterium necrophorum Abscess of Vastus Lateralis. J EMERG MED. 2016;50(4):e191-3.

7. Miyamoto S, Toi T, Kotani R, Iwakami T, Yamada S, Nishido H, Suzuki Y, Ishikawa H, Murakami M, Hoya K. Lemierre Syndrome Associated with Ipsilateral Recurrent Laryngeal Nerve Palsy: A Case Report and Review. NMC Case Rep J. 2016;3(3):53-7.

8. Schubert AD, Hotz MA, Caversaccio MD, Arnold A. Septic thrombosis of the internal jugular vein: Lemierre's syndrome revisited. LARYNGOSCOPE. 2015;125(4):863-8.

9. Johannesen KM, Bodtger U. Lemierre's syndrome: current perspectives on diagnosis and management. INFECT DRUG RESIST. 2016;9:221-7.

10. Jariwala RH, Srialluri S, Huang MZ, Boppana SB. Methicillin-resistant Staphylococcus aureus as a Cause of Lemierre's Syndrome. PEDIATR INFECT DIS J. 2017;36(4):429-31. 
11. Nygren D, Holm K. Invasive infections with Fusobacterium necrophorum including Lemierre's syndrome: an 8-year Swedish nationwide retrospective study. Clin Microbiol Infect. 2020;26(8):10879.

12. Gore MR. Lemierre Syndrome: A Meta-analysis. Int Arch Otorhinolaryngol. 2020;24(3):e379-85.

13. Valerio L, Zane F, Sacco C, Granziera S, Nicoletti T, Russo M, Corsi G, Holm K, Hotz MA, Righini C, et al. Patients with Lemierre syndrome have a high risk of new thromboembolic complications, clinical sequelae and death: an analysis of 712 cases. J INTERN MED. 2021;289(3):325-39.

14. Riordan T. Human infection with Fusobacterium necrophorum (Necrobacillosis), with a focus on Lemierre's syndrome. CLIN MICROBIOL REV. 2007;20(4):622-59.

15. Chirinos JA, Lichtstein DM, Garcia J, Tamariz LJ. The evolution of Lemierre syndrome: report of 2 cases and review of the literature. Med (Baltim). 2002;81(6):458-65.

16. Albertyn LE, Alcock MK. Diagnosis of internal jugular vein thrombosis. RADIOLOGY. 1987;162(2):505-8.

17. Charles K, Flinn WR, Neschis DG. Lemierre's syndrome: a potentially fatal complication that may require vascular surgical intervention. J VASC SURG. 2005;42(5):1023-5.

18. Campo F, Fusconi M, Ciotti M, Diso D, Greco A, Cattaneo CG, de Vincentiis M. Antibiotic and Anticoagulation Therapy in Lemierre's Syndrome: Case Report and Review. J Chemother. 2019;31(1):42-8.

19. Chapman R, Tully A. A life-threatening sore throat. LANCET. 2004;364(9428):112.

20. Choi BM, Son SW, Park CK, Lee SH, Yoon HK. Extensive Bilateral Lemierre Syndrome due to Methicillin-Resistant Staphylococcus epidermidis in a Patient with Lung Adenocarcinoma. Tuberc Respir Dis (Seoul). 2015;78(3):289-92.

21. Johannesen KM, Bodtger U. Lemierre's syndrome: current perspectives on diagnosis and management. INFECT DRUG RESIST. 2016;9:221-7.

22. Phan T, So TY. Use of anticoagulation therapy for jugular vein thrombus in pediatric patients with Lemierre's syndrome. Int J Clin Pharm. 2012;34(6):818-21.

23. Lakshminarayana PH, Woodske ME: A unique case of lemierre syndrome associated with thrombophilia in an adult and the role of anticoagulation. Case Rep Med 2010, 2010.

24. Schmid T, Miskin H, Schlesinger Y, Argaman Z, Kleid D. Respiratory failure and hypercoagulability in a toddler with Lemierre's syndrome. PEDIATRICS. 2005;115(5):e620-2.

\section{Figures}




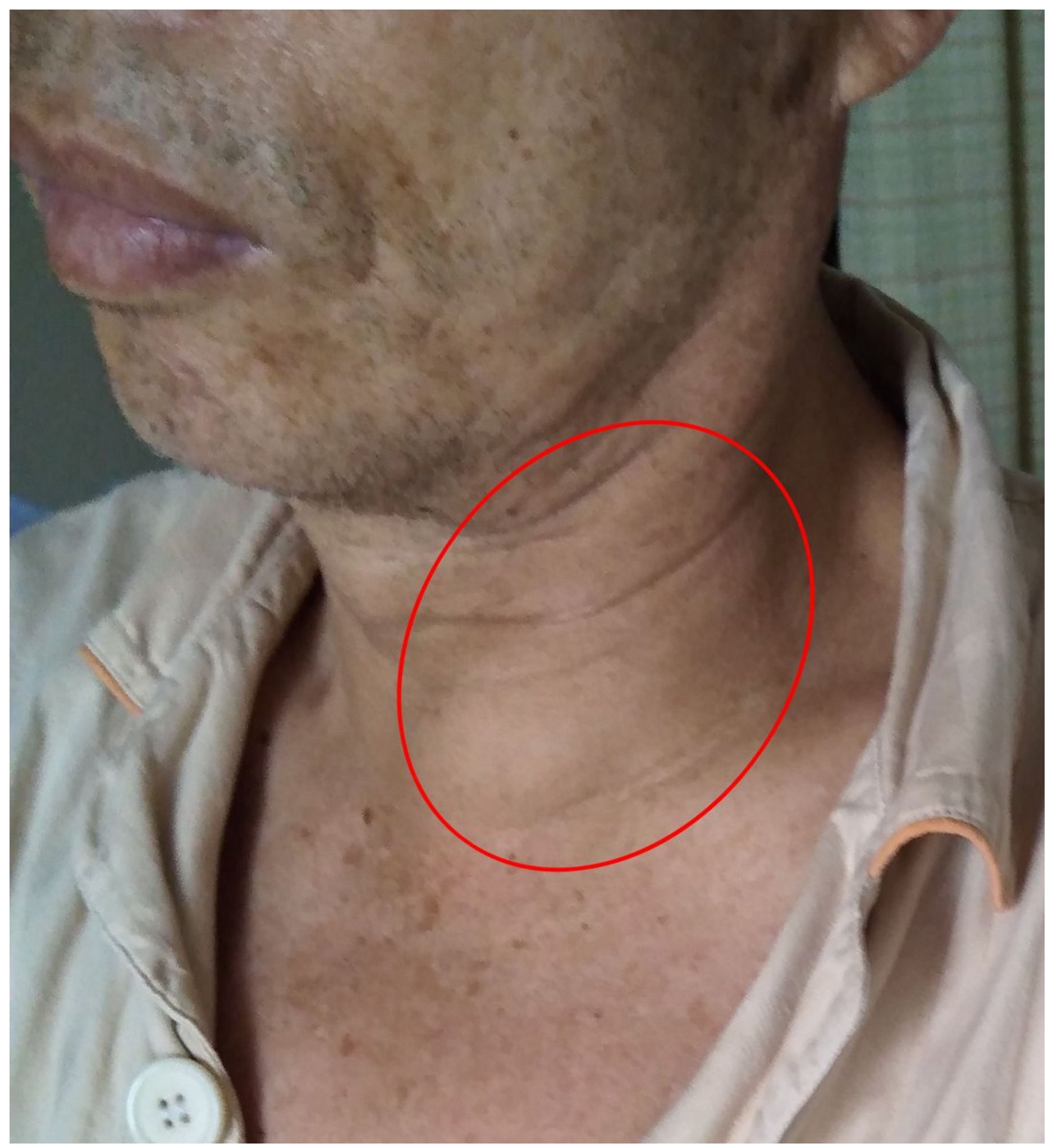

Figure 1

Picture of the patient's neck showed a huge mass with tenderness. 
A
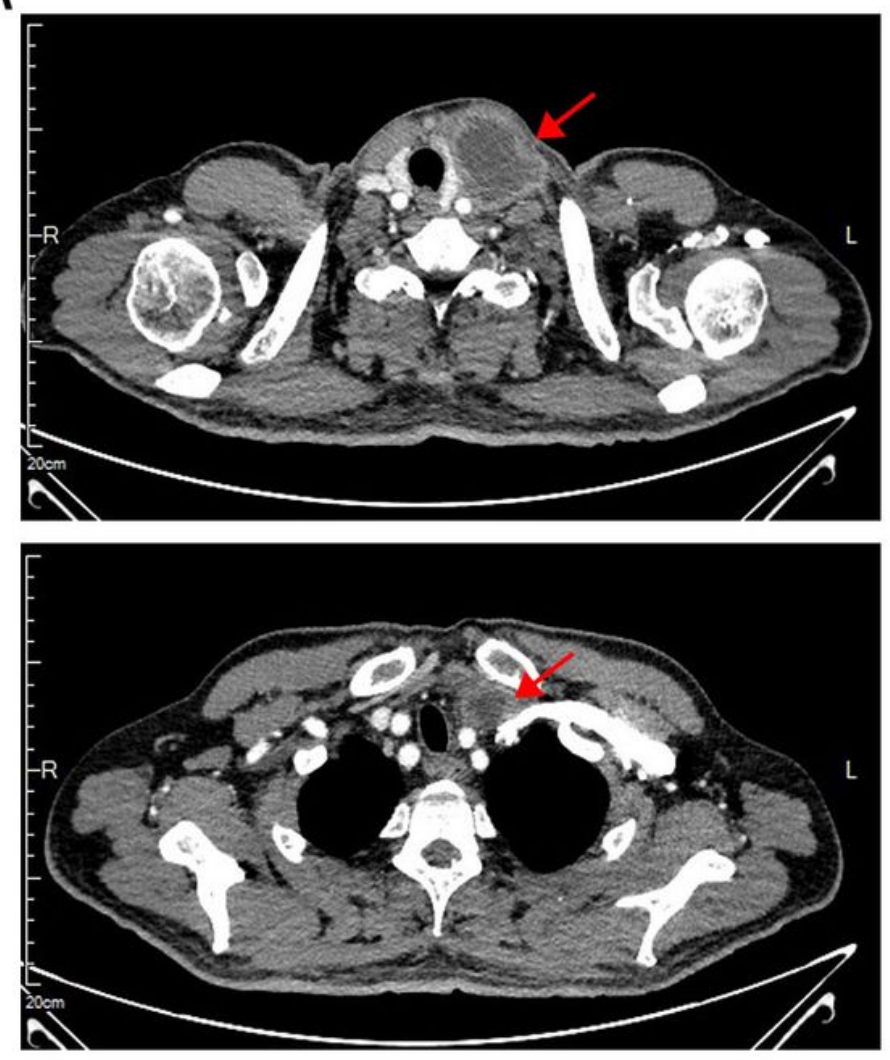

B

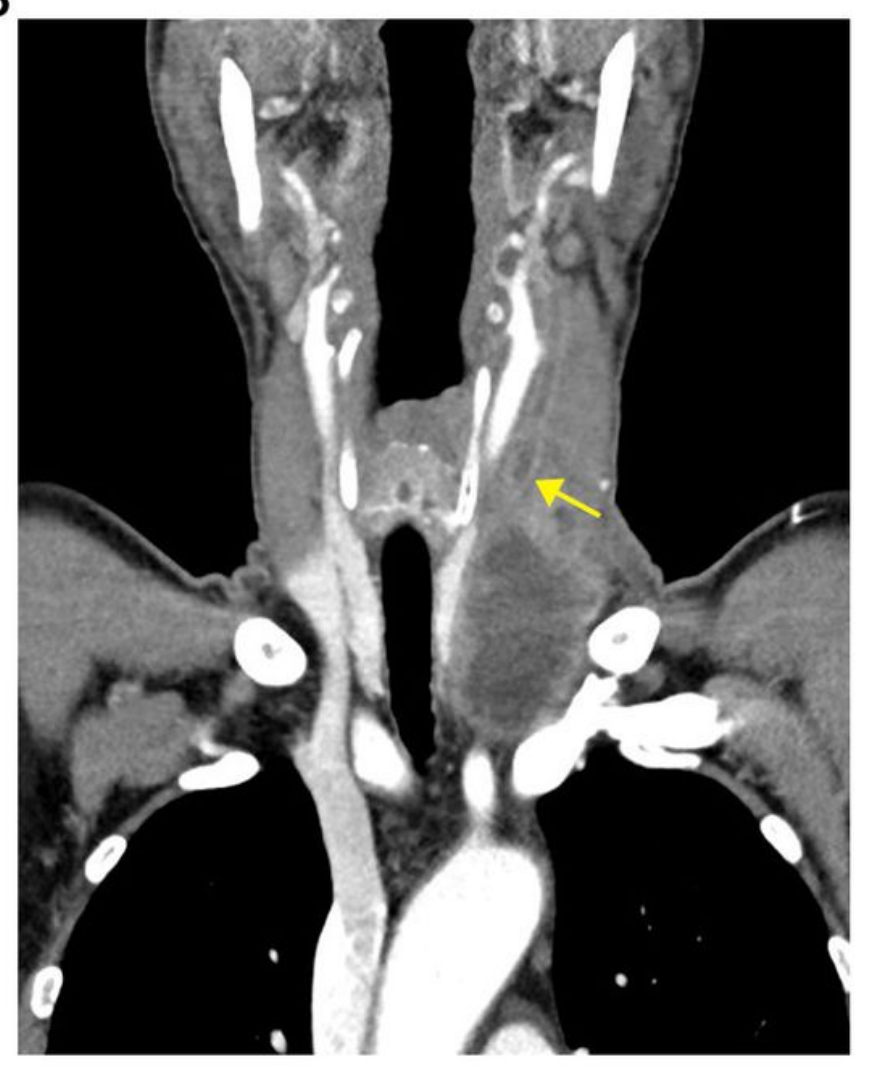

\section{Figure 2}

(A) Coronal plane of CT showed neck mass spread down to superior mediastinum with iquefactive necrosis in the center (red arrows). Trachea was oppressed to the right side. (B) Significant intraluminal filling defect and thrombosis was found in internal jugular vein (yellow arrow). 


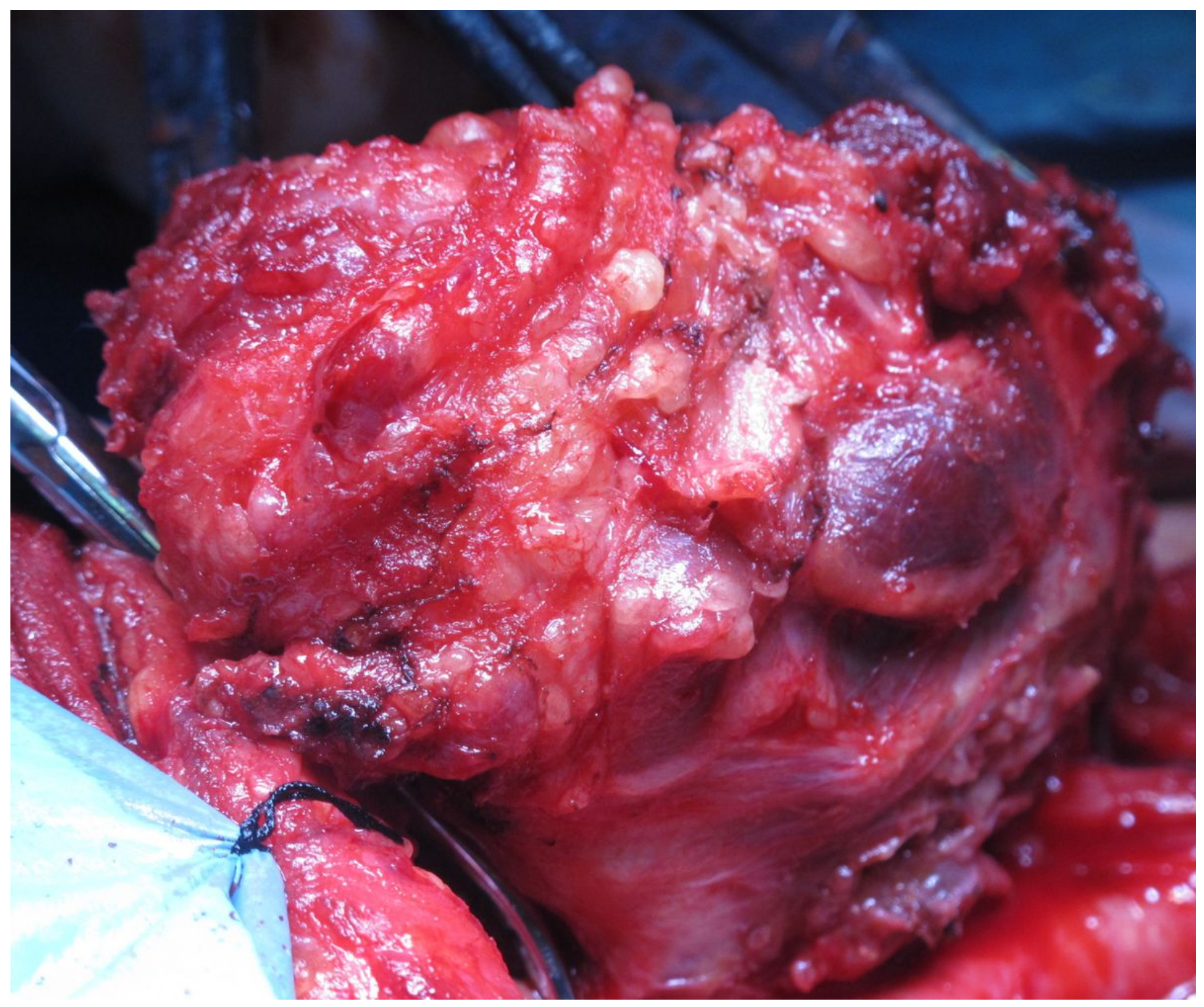

\section{Figure 3}

Intraoperative view showed ligation of IJV and separation of the mass. 


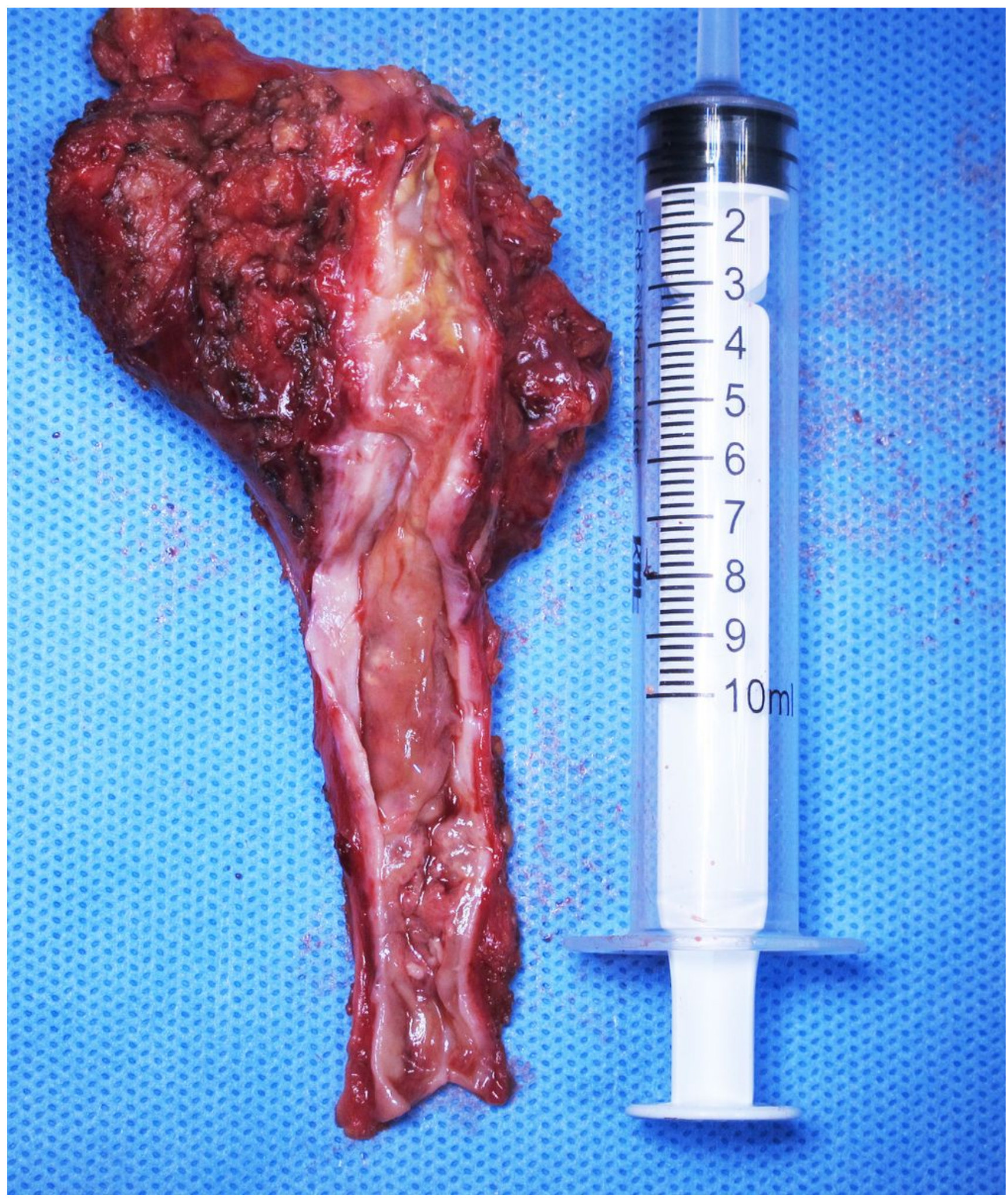

Figure 4

The resected neck mass as well as left IJV was showed. Intraluminal thrombosis could be seen clearly when opening the internal jugular vein. 

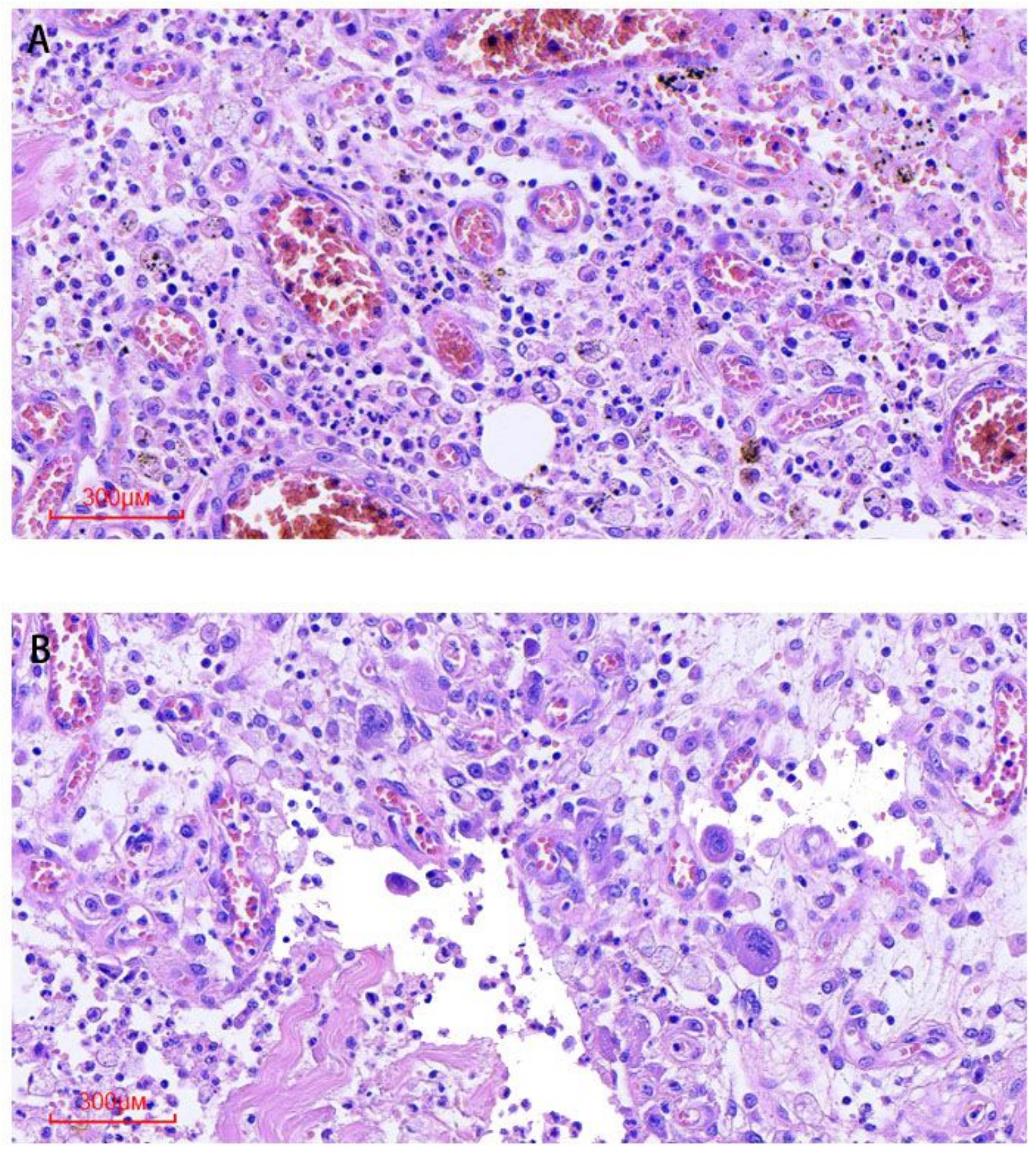

Figure 5

Representative pathological photomicrograph demonstrated thrombus (A) and abscess formation (B). 